\title{
Antimicrobial activity of synthetic chalcone-derived molecules in mixed biofilms and in vitro and in vivo toxicity
}

\author{
Joice Graciani, Pedro Luiz Rosalen, Emmanuely de Oliveira C. dos Santos, Mayara A. Rocha Garcia, Josy \\ Goldoni Lazarini, Luís Octávio Regasini, Janaina de Cássia Orlandi Sardi
}

\section{Resumo}

The microorganisms resulting from a pulp infection produce toxic products that are responsible for the persistence and development of endodontic apical periodontitis, with the most recurrent bacterium, Enterococcus faecalis and yeast Candida albicans. Thus, the search for new pharmacological approaches is necessary so that the intracanal medicine associated with mechanical cleaning is able to totally eliminate these pathogens. Chalcone is a flavonoid that has antimicrobial properties and has become the target of studies for antibacterial and antifungal activity. The objective of this project was the bioprospection of aminochalcones against C. albicans and E. faecalis planktonic, as well as biofilms of $\mathrm{C}$. albicans and $\mathrm{E}$. faecalis (mono and mixed), human gingival fibroblast (FGH) toxicity (in vitro) and in Galleria mellonella (in vivo) of chalcona with better activity.

\section{Palavras-chave: \\ Mixed biofilm, aminochalcone, toxicity.}

\section{Introduction}

Pulp infection can result in the microbial colonization of the entire root canal system. The failures of endodontic treatments are associated, in part, to the presence of a resistant microbiota, being the most recurrent bacterium, $E$. faecalis and yeast $C$. albicans the main fungus isolated from the root canals.

The study of synthetic chalcones, in which functional groups are introduced, has been widely carried out with the aim of increasing these activities. This compound is easily found in various types of plants, including fruits and vegetables and has known biological activities, such as anti-inflammatory, antipyretic and analgesic.

The objective was to prospect 30 chalcones modified against $C$. albicans and $E$. faecalis through the study of Minimal Inhibitory Concentration (MIC) and Minimum Fungicide Concentration and Minimum Bactericidal Concentration (MFC / MBC), in addition to the biofilm study of $C$. albicans and $E$. faecalis and to determine in vivo toxicity using the $G$. mellonella invertebrate model.

\section{Results and Discussion}

Results of cellular viability of I-38 treated biofilms, in addition to in vivo toxicity studies using the Galleria mellonella invertebrate model, demonstrated that I-38 was able to inhibit the growth of $E$. faecalis and $C$. albicans planktonic cells with MIC and MFC values between $15.6 \mu \mathrm{g} / \mathrm{mL}$ and $7.8 \mu \mathrm{g} / \mathrm{mL}$, respectively. In monkeys in formation, concentrations of $1 \mathrm{x}$ MIC and $10 \mathrm{x}$ MIC of I-38 reduced $1 \log _{10}$ and totally inhibited $C$. albicans, respectively. E. faecalis was reduced in $1 \log _{10}$ when treated with $1 \times$ MIC and $2 \log _{10}$ with the use of $10 x$ MIC, whereas in the mature biofilm there was also a reduction in the amount of $C$. albicans $2 \log _{10}$ when using $1 \mathrm{x} \mathrm{MIC}$ and $4 \log _{10}$ in $10 \mathrm{x}$ MIC and $E$. faecalis $0.5 \log _{10}$ when using $1 x$ MIC and $2.5 \log _{10}$ to $10 x$ MIC. In mixed biofilms in formation, concentrations of $1 \mathrm{x}$ MIC and 10x MIC of $1-38$ reduced $C$. albicans at $4 \log _{10}$ and total inhibition, respectively. E. faecalis was reduced by 1 $\log _{10}$ when treated with $1 \times$ MIC and $8 \log _{10}$ when $10 x$ MIC was used. In relation to the mixed mature biofilm of $C$. albicans and $E$. faecalis, there was reduction both $C$. albicans, $2.5 \log _{10}$ when using $1 \times$ MIC and $4.5 \log _{10} 10 \mathrm{X}$ MIC, as in $E$. faecalis $2 \log _{10}$ when using $1 x$ MIC and 6 $\log _{10} 10 x$ MIC. In order to verify the acute toxicity in vivo, tests were used on $G$. mellonella. It was found that in all groups survival was $100 \%$ during the 96 hours of observation, as can be seen in figure 1 .

Figure 1. In vivo systemic toxicity of compound I-38 in G. mellonella larvae. The compound showed no toxic effects at the concentrations used.

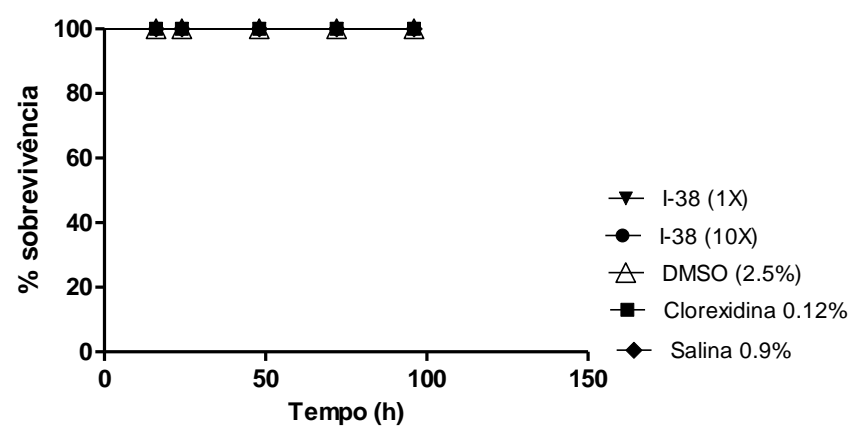

\section{Conclusion}

I-38 presented potent antimicrobial activity on Candida albicans and Enterococcus faecalis, in addition to presenting a low in vivo toxicity, being able to be proposed as an alternative agent in the control of biofilm dependent diseases.

\section{Acknowledgment}

I thank my supervisor Janaína C.O. Sardi, my friend Emmanuely dos Santos. CNPq for the granting of the Scientific Initiation Grant.

\footnotetext{
${ }^{1}$ Gow N.; Yadav B. Microbe Profile: Candida albicans: a shape-changing, opportunistic pathogenic fungus of humans. Microbiology. 2017 Aug; 15

${ }^{2}$ Seneviratne CJ, Wang Y, Jin L, Abiko Y, Samaranayake LP. Candida albicans biofilm formation is associated with increased anti-oxidative capacities. Proteomics. 2008 Jul; 8.

${ }^{3}$ Paganini ER. Síntese e atividade anti-HCV, anti-Candida e antibacteriana de nitrochalconas. 2016 Mar; 11
} 